\title{
Entre el avance y las barreras. Enfoques de igualdad de género en la política pública de mujeres rurales en Colombia
}

\author{
Between Gains and the Barriers. Approaches to Gender Equality \\ in the Public Policy of Rural Women in Colombia \\ Entre o avanço e as barreiras. Enfoques de igualdade de gênero \\ na política pública de mulheres rurais na Colômbia
}

\section{María Mónica Parada HernándeZ}

FECHA DE RECEPCIÓN: 10 DE AGOSTO DE 2017. FECHA DE APROBACIÓN: 15 DE MARZO DE 2018

DOI: http://dx.doi.org/10.12804/revistas.urosario.edu.co/sociojuridicos/a.6612

Para citar este artículo: Parada Hernández, M. M. (2018). Entre el avance y las barreras. Enfoques de igualdad de género en la política pública de mujeres rurales en Colombia. Estuios Socio-Jurídicos, 20(2), 103-128. doi: http://dx.doi. org/10.12804/revistas.urosario.edu.co/sociojuridicos/a.6612

\section{RESUMEN}

En 2002 se promulgó la Ley de Mujeres Rurales en Colombia como resultado de las demandas de mujeres campesinas, indígenas y afrocolombianas por su reconocimiento como sujetos autónomos y su inclusión en los procesos de diseño e implementación de las políticas de desarrollo rural del país. Tras dieciséis años, el Estado colombiano se ha visto incapacitado, no solo para cumplir con las disposiciones de la ley, sino para abordar las desigualdades de género que afectan en particular a las mujeres rurales. La explicación que propongo es que, a pesar de que existe una preocupación por incorporar los temas de género en la agenda pública de desarrollo rural, el enfoque que ha prevalecido hasta el momento es el de la acción positiva que limita las discusiones sobre los sistemas y procesos que producen y reproducen las desigualdades de género.

Palabras clave: transversalización de género, desarrollo rural, política agraria, mujeres rurales, acción positiva.

\section{ABTSRACT}

In 2002, Colombia adopted the Rural Women's Law as a result of the demands of peasant, indigenous and Afro-Colombian women for their recognition as autonomous subjects and their inclusion in the processes of design and implementation of the rural development policies. After 16 years, the Colombian state has been unable not only to comply with the provisions of the law, but also to address the gender inequalities that affect rural women.

* Abogada y socióloga de la Universidad del Rosario. Estudiante de maestría en Derecho Público de la misma universidad. Investigadora del Observatorio de Restitución y Regulación de Derechos de Propiedad Agraria. Correo electrónico: monicaparadaher@gmail.com 
The explanation I propose is that, although there is a concern to incorporate gender issues in the public agenda of rural development, the approach that has prevailed so far is that of positive action that limits the discussions about the systems and processes that produce and reproduce gender inequalities.

Keywords: gender mainstreaming, rural development, agrarian public policy, rural women, positive action.

\section{RESUMO}

Em 2002 se promulgou a Lei de Mulheres Rurais na Colômbia como resultado das demandas de mulheres camponesas, indígenas e afro-colombianas pelo reconhecimento como sujeitos autónomos e sua inclusão nos processos de desenho e implementação das políticas de desenvolvimento rural do país. Depois de 16 anos, o estado colombiano se tem visto incapacitado não só para cumprir com as disposições da lei, mas também para abordar as desigualdades de gênero que afetam particularmente às mulheres rurais. A explicação que proponho é que, a pesar de que existe uma preocupação por incorporar os temas de gênero na agenda pública de desenvolvimento rural, o enfoque que tem prevalecido até o momento é o da ação positiva que limita as discussões sobre os sistemas e processos que produzem e reproduzem as desigualdades de gênero.

Palavras- chave: integração de gênero, desenvolvimento rural, política agrária, mulheres rurais, ação positiva 


\section{Introducción}

El surgimiento de políticas públicas y de desarrollos normativos con enfoque de género son el resultado de la adopción de una agenda internacional fundada en la promoción de la equidad de género y en el reconocimiento de la importancia de las mujeres para el desarrollo, en especial en los países más pobres. Este proceso se enmarca dentro de lo que en la agenda internacional se conoce como transversalización de género (gender mainstreaming).

El presente artículo hace parte de una investigación más amplia que tiene como objetivo indagar por la forma en la que el Estado colombiano ha abordado las desigualdades que afectan en particular a las mujeres rurales. Los resultados que se presentan aquí muestran que la incorporación de los temas de género en la agenda pública de desarrollo rural en Colombia ha estado limitada por la adopción de un enfoque de acción positiva, orientado a crear mecanismos específicos para las mujeres rurales, y con ello se ha obviado la discusión frente a los sistemas y procesos que producen y reproducen las desigualdades de género.

Propongo un análisis de las normas jurídicas y de los documentos de política que se han expedido para beneficiar a las mujeres rurales, desde 1984 hasta 2016, año de la firma del acuerdo de paz entre el gobierno de Colombia y la guerrilla de las FARC. Este acuerdo marca un momento clave en la adopción de los compromisos de género del Estado colombiano frente a las mujeres rurales mediante la promesa de aplicar un enfoque transversal de género en los diseños de política para el escenario del posacuerdo. Un análisis desde la perspectiva normativa y de política pública permite develar la forma en la que el Estado y otros actores sociales, que negocian en el campo jurídico y político, se aproximan a los problemas de las desigualdades en razón del género.

Asimismo, se utilizan documentos provenientes de organizaciones sociales y de la cooperación internacional que se han encargado de identificar las barreras de acceso de las mujeres rurales a las instituciones. Por último, se utiliza información oficial conseguida a través de documentos de política pública, derechos de petición y entrevistas semiestructuradas a actores clave en las agencias del Estado cuya misión es promover la equidad de género en Colombia (Consejería Presidencial para la Equidad 
de la Mujer y Dirección de Mujer Rural del Ministerio de Agricultura y Desarrollo Rural).

El texto está estructurado de la siguiente manera. Primero, se aborda la pregunta acerca de cómo los Estados se aproximan a los problemas de inequidad de género a través de tres enfoques que se han adoptado y cuáles son las herramientas utilizadas. Posteriormente, se identifican las principales características y barreras del enfoque de transversalización de género para luego abordarlas desde la política pública de las mujeres rurales. Finalmente, se hacen unas reflexiones al respecto.

\section{Cómo aborda el Estado las desigualdades de género}

La pregunta por cómo operan desigualdades de género conduce a pensar en la forma en la que un Estado distribuye y orienta sus recursos para abordar el problema. En otras palabras, se trata de entender cómo concibe el Estado el problema de las desigualdades entre hombres y mujeres y, una vez reconocidas, qué mecanismos utiliza para (re)distribuir recursos en aras de disminuir dichas desigualdades.

Un Estado formula una serie de objetivos y desarrolla estrategias para cumplirlos (Skocpol, 1985). Estos objetivos pueden derivarse de: (i) el carácter autónomo del Estado que lo lleva a buscar las transformaciones, incluso si estas generan resistencias por parte de las élites políticas y económicas; o (ii) de las demandas de sectores sociales determinados. Independientemente del origen de dichos objetivos, una vez formulados, el Estado está en la necesidad de dotarse de las herramientas necesarias para cumplirlos (Mann, 2004; Skopcol, 1985; Acemoglu, et al., 2011).

Disminuir las desigualdades entre hombres y mujeres se ha convertido en un objetivo de política tanto para los Estados nación como para las organizaciones internacionales. Estas últimas, junto con los movimientos feministas y de mujeres, han sido de gran importancia en el establecimiento de pautas para abordar el problema (True \& Mintrom, 2001; Hafner-Burton \& Pollack, 2002).

Esta preocupación se puede rastrear a la Década de la Mujer (19751985), periodo en el cual se adoptó la Convención sobre la Eliminación de Todas las Formas de Discriminación contra la Mujer (en adelante CEDAW) 
de 1979, mediante la cual los Estados parte se obligaron a tomar "todas las medidas apropiadas, incluso de carácter legislativo, para asegurar el pleno desarrollo y adelanto de la mujer, con el objeto de garantizarle el ejercicio y el goce de los derechos humanos y las libertades fundamentales en igualdad de condiciones con el hombre" (Artículo 3). Esta convención marcó el inicio de la incorporación de los temas de género en la agenda pública de los Estados y de las organizaciones internacionales, proceso que llegó a su auge la década de los noventa con las estrategias promovidas por la Unión Europea y con la firma de la Declaración y Plataforma de Acción de Beijing, en 1995.

Estos instrumentos contienen diferentes objetivos encaminados a aumentar la participación de las mujeres en todas las esferas de la vida social, y compromete a los Estados a impulsar estrategias para alcanzar dichos objetivos. Sin embargo, dependiendo de contextos específicos, distintos enfoques han sido aplicados para abordar las desigualdades de género. Estos han sido agrupados por la literatura internacional en tres categorías (Rees, 1998; Walby, 2005): (a) igualdad de oportunidades; (b) acción positiva; (c) transversalización de género (ver tabla 1). Los tres enfoques, que hoy en día coexisten, han sido producto de momentos históricos específicos, aunque hay quienes afirman que la transversalización de género es la síntesis de los otros dos (Squires, 2005).

A continuación, propongo identificar, a través de la revisión de literatura internacional, cuáles son los elementos que caracterizan a cada uno de los enfoques, y resaltar sus límites y potencialidades.

Tabla 1. Características de los tres enfoques

\begin{tabular}{|l|l|l|l|}
\hline \multicolumn{1}{|c|}{ Enfoque } & \multicolumn{1}{|c|}{ Objetivos } & \multicolumn{1}{c|}{ Ventajas } & \multicolumn{1}{c|}{ Desventajas } \\
\hline $\begin{array}{l}\text { Igualdad } \\
\text { de oportu- } \\
\text { nidades }\end{array}$ & $\begin{array}{l}\text { Modificación de las } \\
\text { legislaciones buscando } \\
\text { la igualdad formal. }\end{array}$ & $\begin{array}{l}\text { Previene la exclusión } \\
\text { de un grupo de ciertas } \\
\text { esferas. } \\
\text { Oportunidades para } \\
\text { la participación de un } \\
\text { grupo. }\end{array}$ & $\begin{array}{l}\text { Se incluye a las mujeres en } \\
\text { la medida en que se des- } \\
\text { empeñen bajo estándares } \\
\text { masculinos. }\end{array}$ \\
$\begin{array}{l}\text { Ausencia de análisis de esfe- } \\
\text { ras público-privadas. } \\
\text { Solo beneficia a cierto tipo } \\
\text { de mujeres. }\end{array}$ \\
\hline
\end{tabular}

Continúa 


\begin{tabular}{|l|l|l|l|}
\hline \multicolumn{1}{|c|}{ Enfoque } & \multicolumn{1}{|c|}{ Objetivos } & \multicolumn{1}{c|}{ Ventajas } & \multicolumn{1}{c|}{ Desventajas } \\
\hline $\begin{array}{l}\text { Acción } \\
\text { positiva }\end{array}$ & $\begin{array}{l}\text { Reconocen la diferen- } \\
\text { cia como punto de } \\
\text { partida para incorporar } \\
\text { a favor de un grupo } \\
\text { poblacional específico. }\end{array}$ & $\begin{array}{l}\text { Objetivos claros y me- } \\
\text { dibles. } \\
\text { Énfasis en la igualdad en } \\
\text { el resultado. } \\
\text { Identifica barreras de } \\
\text { acceso. }\end{array}$ & $\begin{array}{l}\text { Mujeres entran a competir } \\
\text { en campos de dominación } \\
\text { masculina. } \\
\text { No transforma condiciones } \\
\text { de segregación. }\end{array}$ \\
\hline $\begin{array}{l}\text { Transversa- } \\
\text { lización de } \\
\text { género }\end{array}$ & $\begin{array}{l}\text { Se enfoca en los siste- } \\
\text { mas, procesos y nor- } \\
\text { mas que generan las } \\
\text { desigualdades. }\end{array}$ & $\begin{array}{l}\text { Desafía la "neutralidad" } \\
\text { de las políticas. } \\
\text { Reconoce y responde a a } \\
\text { la diversidad. }\end{array}$ & $\begin{array}{l}\text { Aplicación indiscriminada y } \\
\text { no contextualizada. } \\
\text { Ausencia de un análisis de } \\
\text { las desigualdades estructura- } \\
\text { les de género. }\end{array}$ \\
\hline
\end{tabular}

Fuente: Elaboración propia con base en Rees (1998), Walby (2005) y Squires (2005).

\section{Enfoque de igualdad de oportunidades}

Este enfoque tiene sus antecedentes en los movimientos que impulsaron el reconocimiento de derechos políticos y civiles en el marco de la Revolución francesa -ver por ejemplo (Gouges, 1791) - y que luego fue apropiado por las corrientes feministas del liberalismo clásico (Wollstonecraft, 1972). Tiene como objetivo principal que las mujeres sean reconocidas por el Estado y la sociedad en iguales términos que los hombres. Por ello apela a la trasformación de los sistemas normativos, ya sea por vía legal o jurisprudencial, que permita una inclusión formal de las mujeres u otros grupos históricamente discriminados. El uso de este enfoque ha permitido a las mujeres el acceso a derechos políticos (auspiciado por los movimientos sufragistas a finales del siglo XIX y principios del siglo XX) o el reconocimiento de su autonomía en la administración de sus patrimonios ${ }^{1}$ (Jaramillo, 2000).

A pesar de los avances que la aplicación del enfoque de igualdad de oportunidades ha promovido, su alcance es limitado por varias razones. La principal crítica es que la inclusión de las mujeres está mediada por estándares masculinos. En otras palabras, este enfoque carece de un análisis acerca de las relaciones entre hombres y mujeres, y de la relación que existe entre lo que se considera como el ámbito "público" (roles

1 En Colombia se reconoció la igualdad jurídica de los hombres y las mujeres con el Decreto 2820 de 1974 que modifica el Código Civil de 1887. 
asociados a lo masculino, como el ejercicio de la política) y el "privado" (roles asociados a lo femenino, como la economía del cuidado) ${ }^{2}$ (Guerrina, 2002). Además, la promoción de leyes de igualdad, que son el principal instrumento para implementar este enfoque, tiende a esencializar las experiencias de las mujeres (Smart, 2002) y carece de un enfoque interseccional (Mouritz Marfelt, 2006).

Como resultado, solo aquellas mujeres que cuentan con determinados tipos de capital y viven en contextos que les permiten un ejercicio más o menos autónomo de sus derechos resultan beneficiadas por los mecanismos de igualdad de oportunidades. Ejemplo de ello son las condiciones de inequidad en la tenencia de la tierra de las mujeres rurales en África y Latinoamérica, lugares donde, a pesar del reconocimiento legal, el ejercicio del derecho de propiedad se encuentra frenado por las lógicas propias de lo rural y por los sistemas de tenencia informales soportados por la costumbre (Walker, 2003; Mwagiru, 2001; Pasura, 2010).

De ello se desprende que el enfoque sea insuficiente para sociedades con profundas desigualdades sociales en las que un reconocimiento meramente formal no es garantía de acceso de todas las mujeres a bienes y servicios promovidos o protegidos socialmente.

\section{Enfoque de acción positiva}

La discriminación positiva se basa en la noción de que la pertenencia a un grupo determinado (mujeres, grupos étnicos, grupos LGBTI) incide en el reconocimiento (o ausencia de reconocimiento) de derechos y en el acceso a bienes y servicios. Esto conlleva a que se planteen mecanismos que faciliten el acceso de las mujeres a dichos recursos y a que sean compensadas por las condiciones que permiten la desigualdad (Rees, 1998).

Esta estrategia tiene una ventaja frente a la anterior, pues parte de la necesidad de identificar las barreras de acceso de las mujeres a distintos recursos. Por eso ha sido fundamental en la garantía de derechos de

2 La discusión sobre la dicotomía público-privado ha sido superada desde la teoría feminista (Hanisch, 2016; Millet, 1995). Sin embargo, estas distinciones siguen operando en el ámbito de la política pública, por ejemplo, cuando los Estados se niegan a regular las actividades asociadas a la economía del cuidado que son tradicionalmente consideradas como femeninas o de la esfera privada de la familia. 
grupos históricamente discriminados al convertirse en un mecanismo para equiparar las desigualdades sociales (Durango Álvarez, 2016), reparar las injusticias históricas y la discriminación estructural, y crear diversidad en la representación proporcional de los grupos (Murillo Martínez, 2010).

Para abordar el problema de la equidad de género, la acción positiva opta por reconocer las diferencias sociales que existen entre hombres y mujeres, y sobre ellas diseñar mecanismos que eliminen las barreras o garanticen unas condiciones mínimas para que las mujeres accedan a la distribución de recursos en materia educativa, laboral, crediticia y de participación, entre otros.

El ejemplo por excelencia de este tipo de medidas son las normas que establecen cuotas electorales para garantizar que las mujeres tengan representación en los órganos estatales de toma de decisión. Al respecto Dahlerup (2006) presenta una serie de estudios comparativos de países en los que las leyes de cuotas se han impuesto y muestra sus distintos desenlaces. Mientras que en algunos la participación de las mujeres en órganos como el parlamento ha permitido transformar las agendas políticas en los distintos niveles de la administración, en otros en los que prevalecen sistemas de género tradicionales o en aquellos que cuentan con órganos parlamentarios compuestos en su mayoría por hombres, las cuotas son una inclusión apenas simbólica sin efectos concretos sobre la vida de las mujeres.

Es así como las estrategias de acción positiva también tienen alcances limitados en tanto que su objetivo no es transformar las estructuras de las organizaciones y los patrones culturales que reproducen las inequidades de género (Rees, 1998; Saldarriaga Grisales \& Ramírez Monsalve, 2015; Morris, 2005); por el contrario, se presentan como medidas a corto plazo que sirven para apoyar la transición a sociedades más igualitarias. Además, se ha criticado que cuando el derecho protege los intereses específicos de las mujeres a través de las normas de acción positiva, por ejemplo, ha generado efectos adversos a los conseguidos, precisamente por la permanencia de un sistema de valores masculinos que definen cómo deben ser tratados los temas de mujeres (Jaramillo, 2009). 


\section{Enfoque de la transversalización de género}

El enfoque de transversalización de género se presenta como una estrategia a largo plazo que busca transformar las instituciones, los sistemas y las organizaciones, a partir del reconocimiento de su carácter androcéntrico que favorece a los hombres por encima de las mujeres. Requiere de una mirada crítica sobre las prácticas que se presentan como neutrales, en términos de género, y que aparentan garantizar la igualdad para todas las personas cuando en realidad comportan mecanismos de exclusión para las mujeres (Rees, 1998). Por esta razón hay quienes consideran que la transversalización de género es una respuesta y una reacción a un periodo anterior de intervenciones dirigidas a mujeres, marcado por estrategias de igualdad de oportunidades y acción positiva, apuesta que resultó insuficiente debido a su carácter marginal en la formulación de políticas (Mehra \& Gupta, 2006).

Hoy en día, se entiende por transversalización de género una estrategia para evaluar las implicaciones que tiene cualquier acción planificada del Estado, incluyendo la legislación, en la vida de hombres y mujeres. $\mathrm{Su}$ finalidad es la de incluir las preocupaciones y experiencias de mujeres y hombres en del diseño, implementación, monitoreo y evaluación de políticas y de programas en todas las esferas políticas, económicas y sociales para que las mujeres y los hombres se beneficien por igual y la desigualdad no se perpetúe (Resolución 1997/2 ECOSOC).

Las políticas públicas desarrolladas sobre este enfoque deben reconocer y responder a la diversidad; de ahí que también se haya utilizado para temas asociados con políticas antiracistas o de discapacidad (Squires, 2005; Klein, 2012; Hankivsky, 2005) aunque su desarrollo teórico y práctico ha sido más abordado desde la categoría de género. Así, reconocer la diferencia implica renunciar a la idea de incluir a las mujeres en las instituciones y prácticas creadas por los hombres (Cockburn, 1991) y, por el contrario, apostar por una verdadera transformación de las mismas.

A pesar de los lineamientos propuestos desde los organismos internacionales, la transversalización de género ha sido extremadamente difícil de poner en operación (Mazey, 2002). De acuerdo con Daly (2005), esto se debe a que, en la práctica, el enfoque: primero, sigue sin incorporar un análisis de género de las desigualdades consideradas como estructurales; 
segundo, responde a la compulsión de los Estados de mejorar la política de género como una manera de modernizarse sin abrir el debate sobre la desigualdad de género como un problema estructural; tercero, a que existe una necesidad permanente de satisfacer las exigencias de los organismos que proveen recursos para la cooperación; y cuarto, a que está movido por la necesidad satisfacer otros objetivos que no están directamente relacionados con la igualdad de género, por ejemplo, el de la productividad (ver por ejemplo los estudios de Kabeer, 2003).

Las evaluaciones disponibles, en especial en países de la Unión Europea (Beveridge \& Nott, 2002), sugieren que el principal problema es la aplicación del enfoque sin tener en cuenta las particularidades de los contextos (Moser \& Moser, 2005; Clisby \& Enderstein, 2017). Además, se ha argumentado que las herramientas utilizadas para desplegar la estrategia varían de acuerdo a los contextos institucionales (Yeandre, Booth, E Bennet, 1998) y a los sectores a las que se aplican (salud, educación, etc.) (Kabeer, 2003). En otras palabras, cada sector debe aplicar estartegias específicas que obedezcan a las necesidades de su contexto, lo que hace más difícil concretar las rutas de acción adecuadas y delimitar el contenido de la trasnversalización de género.

Preocupa entonces que, a pesar de existir una idea generalizada sobre qué es la transversalización de género ${ }^{3}$, nadie está seguro de qué se requiere para llevarla a la práctica (Beveridge $\varepsilon$ Nott, 2002). Sin embargo, la literatura internacional ha señalado algunos de los mecanismos que deben incluirse en los diseños de política pública, (ver tabla2).

Tabla 2. Mecanismos de transversalización de género

\begin{tabular}{|l|l|}
\hline \multicolumn{1}{|c|}{ Mecanismo } & \multicolumn{1}{c|}{ Descripción } \\
\hline $\begin{array}{l}\text { Transformación de los } \\
\text { sistemas jurídicos }\end{array}$ & $\begin{array}{l}\text { Reformas normativas en las que se garantice la igualdad de } \\
\text { derechos entre hombres y mujeres (enfoque de igualdad de } \\
\text { oportunidades). }\end{array}$ \\
\hline $\begin{array}{l}\text { Medidas de acción positiva } \\
\text { y de discriminación positiva }\end{array}$ & $\begin{array}{l}\text { Medidas específicas para beneficiar a las mujeres o establecer } \\
\text { un sistema de cuotas que garantice la inclusión de las mujeres } \\
\text { en distintas esferas (Meertens, 2016; Rees, 1998). }\end{array}$ \\
\hline
\end{tabular}




\begin{tabular}{|l|l|}
\hline \multicolumn{1}{|c|}{ Mecanismo } & \multicolumn{1}{c|}{ Descripción } \\
\hline $\begin{array}{l}\text { Sistemas de registro } \\
\text { de información }\end{array}$ & $\begin{array}{l}\text { Busca que los sistemas de recolección y registro de información } \\
\text { incluyan variables de género y otras que respondan a unidades de } \\
\text { análisis más pequeñas, como el grupo étnico o la edad (Rees, 1998). }\end{array}$ \\
\hline $\begin{array}{l}\text { Mecanismos de } \\
\text { participación }\end{array}$ & $\begin{array}{l}\text { Asegurar la participación de las mujeres en la toma de deci- } \\
\text { siones a través de mecanismos de consulta en todas las etapas } \\
\text { del desarrollo de políticas públicas para garantizar una amplia } \\
\text { identificación nacional de las estrategias (Keeber, 2003; Walker, } \\
\text { 2003). Asimismo, promover la participación de las mujeres en } \\
\text { los gobiernos local, regional y nacional. }\end{array}$ \\
\hline $\begin{array}{l}\text { Metas e indicadores } \\
\text { de género }\end{array}$ & $\begin{array}{l}\text { Selectividad de métodos, conceptos, herramientas buscando la } \\
\text { tecnocratización del enfoque de género (Squires, 2005). }\end{array}$ \\
\hline Burocracias capacitadas & $\begin{array}{l}\text { Ampliación de la participación de las mujeres en las esferas de } \\
\text { decisión y formación técnica en los distintos niveles de la admi- } \\
\text { nistración (True \& Mintrom, 2001; Squires, 2005; Sañudo, 2015). }\end{array}$ \\
\hline $\begin{array}{l}\text { Presupuestos sensibles } \\
\text { al género }\end{array}$ & $\begin{array}{l}\text { Presupuestos participativos sensibles al género (gender responsive } \\
\text { budgets) en los que se incluyan recursos específicamente desti- } \\
\text { nados para las mujeres (por ejemplo, proyectos de generación } \\
\text { de ingresos para mujeres) o para promover la igualdad de géne- } \\
\text { ro en el sector público (por ejemplo, la capacitación de funcio- } \\
\text { narios en enfoque de género) (Keeber, 2003). }\end{array}$ \\
\hline
\end{tabular}

Fuente: Elaboración propia.

Propongo abordar algunos de estos elementos con el fin de indagar por la forma en la que el Estado colombiano ha buscado resolver los problemas de desigualdad que afectan en particular a las mujeres rurales. La selección de los mismos obedece a la información disponible hasta el momento. Sugiero, entonces, analizar la transformación del sistema jurídico colombiano; la creación de mecanismos de participación para mujeres rurales; la transformación de los mecanismos de registro e información; la instalación de mecanismos de seguimiento y monitoreo de política; y la creación de burocracias especializadas en temas de género. Antes, presento un esbozo de cómo han evolucionado los diseños de política pública en materia de mujeres campesinas/rurales.

\section{Política agraria y transversalización de género en Colombia}

En la década de los ochenta, en un contexto en que los debates internacionales sobre los modelos de desarrollo incluyeron miradas diferenciales 
a los problemas de la pobreza y la desigualdad, Colombia reconoció la importancia de la participación de las mujeres en la producción agrícola, buscando superar la asociación exclusiva de las mujeres rurales con las actividades relacionadas con la economía del cuidado.

La adopción de la CEDAW, incorporada al sistema jurídico colombiano mediante la Ley 051 de 1981, fue el marco de referencia para incluir en la agenda pública "el importante papel que desempeña -la mujer rural- en la supervivencia económica de su familia, incluido su trabajo en los sectores no monetarios de la economía" (CEDAW, Artículo 14). En este contexto el Estado colombiano adoptó la Política Nacional para la Mujer Campesina mediante la cual reconoció los aportes productivos de las mujeres campesinas al desarrollo rural en Colombia y replanteó los programas sociales orientados a su desarrollo, de acuerdo con las realidades de las familias campesinas (Conpes 2109 de 1984). A pesar de los esfuerzos, la política operó de manera deficiente. Esta se formuló de manera paralela a la política de desarrollo rural, lo que dificultó la articulación entre las instituciones encargadas de ejecutar los planes y los programas que afectaban la productividad de las mujeres rurales (Conpes 023 de 1994).

Los reclamos de las mujeres rurales persistieron dado que ninguna de las medidas logró beneficiar, de manera directa, a un grupo significativo de mujeres a pesar de que, en la mayoría de los casos, no se contemplaron medidas abiertamente excluyentes (Deere \& León, 2000). Los movimientos de mujeres continuaron promoviendo una agenda que inclúa la creación de una ley específica para las mujeres rurales y la adopción de la perspectiva de género en la política pública agraria con la esperanza de superar las barreras que impidieron el éxito en el pasado (Mesa de Incidencia Política de las Mujeres Rurales Colombianas, 2015).

Así, en 2002, se expidió la Ley 731 de Mujeres Rurales en la que se consagró la obligación de las entidades del Estado de establecer planes y programas para favorecer a "toda aquella que, sin distingo de ninguna naturaleza e independientemente del lugar donde viva, su actividad productiva esté relacionada directamente con lo rural, incluso si dicha 
actividad no es reconocida por los sistemas de información y medición del Estado o no es remunerada" (Ley 731, Artículo 2) ${ }^{4}$.

En 2011, ante la ausencia de reglamentación de sus disposiciones, el Ministerio de Agricultura creó el Programa de Mujer Rural por medio del cual buscó desarrollar proyectos de emprendimiento productivo, asociactividad y "transversalización de género". Este último intentó incorporar el enfoque a las entidades públicas, territoriales, gremios y al sector privado con componente de formación en perspectiva de género (Vargas \& Villarreal, 2014).

Solo hasta 2012, con la publicación de los Lineamientos de la política pública nacional para la equidad de género (Alta Consejería Presidencial para la Equidad de la Mujer, 2012), se establecieron las disposiciones que darían curso al objetivo de eliminar las inequidades en razón del género. Sería entonces la Consejería Presidencial para la Equidad de la Mujer la encargada de dirigir el proceso de transversalizar el género ${ }^{5}$ en todos los proyectos, programas y/o políticas. A partir de este momento, el desarrollo de las iniciativas para las mujeres rurales se realiza bajo esta sombrilla.

Como se mostrará a continuación, las herramientas utilizadas para incorporar el enfoque en las políticas de desarrollo rural que afectan a las mujeres rurales (y en todas las políticas en general) están apenas en una etapa de diseño. Sin embargo, se revisará la relación que ha tenido el Estado colombiano con las mujeres rurales, desde la década de los ochenta a la actualidad, con el fin de analizar cómo se han abordado algunos de los elementos que la literatura internacional ha señalado como claves para la transversalización de género en los procesos de política pública. Como se indicó, la selección de los elementos obedece ante todo a la información disponible frente al tema.

4 Las implicaciones que tiene crear mecanismos con un enfoque que privilegia las actividades productivas de las mujeres rurales se pueden encontrar en Kabeer (2003).

5 Su adopción implica que "en el diseño e implementación de las políticas, planes y programas sectoriales y territoriales se consideren el efecto diferenciado que tienen las acciones que se realizan y los impactos esperados sobre hombres y mujeres. La transversalización debe estar presente desde la planeación y formulación de proyectos, programas y/o políticas hasta en las formas de valoración de su efectividad en los procesos de monitoreo y evaluación" (Alta Consejería Presidencial para la Equidad de la Mujer, 2012, p. 55). 


\section{Transformación del sistema jurídico y acción positiva}

El derecho es la manifestación más fuerte de poder simbólico (Bourdieu, 1987) y por ello imprime en las relaciones sociales significados determinados por una lógica de dominación masculina. De ahí el interés de transformar los sistemas jurídicos como una forma de incluir a las mujeres.

En Colombia, las normas expedidas a favor de las mujeres rurales (inicialmente nombradas como mujeres campesinas) han sido normas de acción positiva. Es decir, su función no ha sido propiamente la del reconocimiento de la igualdad formal de las mujeres rurales frente a los hombres rurales ${ }^{6}$ (salvo en casos excepcionales), sino que han sido los medios para la creación de medidas específicas que favorecen a las mujeres rurales y les permiten el acceso preferente a determinados recursos (tierra, crédito, proyectos productivos, educación rural, entre otros).

La Ley 135 de 1961 creó un sistema de reforma agraria que privilegió a los hombres en cuanto a la dotación de activos rurales. El Artículo 36 de la ley establecía que "Los varones casados que hayan cumplido diez y ocho (18) años de edad podrán obtener adjudicaciones de baldíos o de unidades agrícolas familiares en colonizaciones o parcelaciones, y contraer, por consiguiente, todas las obligaciones inherentes, sin necesidad de autorización judicial". Esta situación cambió en el año 1988 cuando se expidió la Ley 30 que transformó varias disposiciones de la Ley 135 y permitió que los títulos de propiedad de los predios adjudicados quedaran en cabeza de ambos cónyuges (mecanismo de titulación conjunta). No obstante, esta figura fracasó en tanto que los funcionarios eran renuentes a aplicarla y continuaban privilegiando el prototipo de usuario androcéntrico (Ospina Robledo, 1998) el "núcleo familiar" y la figura del "jefe de hogar" (Deere E León, 2003)

En 1994, el sistema creado por la Ley 135 fue reemplazado por la Ley 160. Esta recogió la titulación conjunta y adicionó disposiciones para priorizar las solicitudes de las jefas de hogar y de mujeres en situación de desprotección a causa del conflicto armado. Estas medidas tuvieron como resultado un aumento de mujeres beneficiarias de la reforma agraria al

\footnotetext{
6 No he hallado registros del uso de "hombres rurales" como una categoría jurídica o de las ciencias sociales.
} 
pasar de un $11 \%$ entre 1961 y 1991 , a un $45 \%$ entre 1995 y 1998 . Sin embargo, otras mujeres quedan excluidas del ámbito de protección de la ley de reforma agraria, sobre todo aquellas que nunca han tendido acceso a la tierra por vía directa ${ }^{7}$; además, los avances ocurrieron de manera lenta y muchas de las barreras a las que se enfrentaron las mujeres rurales para acceder a distintos activos permanecieron intactas (Deere E León, 2003).

Finalmente, en 2002, el Congreso colombiano promulgó la Ley 731 conocida como Ley de Mujer Rural. Esta norma contempló una serie de medidas para eliminar las barreras burocráticas y culturales que impiden el acceso de las mujeres rurales a condiciones dignas de vida a través de la transversalización del enfoque de género en todos los planes y programas de desarrollo rural. Entre otras cosas, la ley estimula el acceso a recursos para inversión, educación, capacitación y recreación; promueve la inclusión de las mujeres en el sistema de seguridad social (por ejemplo insta a la cobertura de estas mujeres en el sistema de riesgos laborales); obliga a las entidades relacionadas con el desarrollo rural, a ajustar procedimientos para promover el acceso de las mujeres a tierra, crédito y proyectos productivos; y fomenta la participación de las mujeres en órganos para la toma de decisiones.

La promulgación de la Ley 731 de 2002 fue un logro importante para los movimientos de mujeres dado que es la primera vez que existe un instrumento normativo de carácter legal que reconoce a las mujeres rurales como sujetos autónomos. Además, constituye un soporte legal para que todas las entidades asociadas con el desarrollo rural incluyan la perspectiva de género en los procedimientos de acceso a fondos, planes y programas que favorezcan actividades rurales. No obstante, como mostraré más adelante, esta norma quedó desprovista de mecanismos para garantizar su cumplimiento.

A pesar de los avances, la institucionalidad agraria que se ha propuesto desde el entramado normativo ha mostrado que el Estado ha imprimido un carácter homogeneizador a las mujeres y no ha tenido en cuenta las diferencias regionales, de clase y étnicas. Así, las políticas se

7 Mujeres que, por ejemplo, no tienen la titularidad de los derechos sobre los predios porque estos están en cabeza de sus compañeros o cónyuges. 
han construido sobre valores tradicionales (Pasura, 2010) y estereotipos alrededor de la agricultura campesina familiar, en la que predomina la figura del jefe de hogar masculino, mientras que las mujeres son vistas como ayudantes (Ospina Robledo, 1998; León \& Deere, 1997), ignorando que la división del trabajo por género tiene variaciones regionales y unas lógicas propias asociadas a lo rural.

Las normas de reconocimiento de derechos y los mecanismos de acción positiva son importantes para garantizar que el enfoque transversal de género se desarrolle. Sin embargo, estas resultan insuficientes cuando no se acompañan de otras medidas que permitan al Estado dotarse de herramientas y recursos para cumplir con las disposiciones normativas. Así las cosas, el reconocimiento que se haga por vía legal corre el riesgo de quedarse en una mera enunciación de derechos, cuando lo que busca la transversalización de género son transformaciones, a largo plazo, de las políticas públicas (Hafner-Burton E Pollack, 2002).

\section{Participación}

La participación es uno de los componentes fundamentales de la transversalización de género pues implica la inclusión de las mujeres en los espacios de decisión en los que se diseña, implementa y evalúan las políticas públicas. Este objetivo se materializa con el reconocimiento político de las mujeres en tanto sujetos autónomos capaces de incidir en el diseño, ejecución y monitoreo de las políticas públicas que las afectan (Sañudo, 2011).

Las transformaciones logradas en las políticas de desarrollo rural para favorecer a las mujeres fueron posibles gracias a la presión de las organizaciones de mujeres y movimientos feministas (León \& Deere, 1997; Bock \& Derkzen, 2012). En Latinoamérica, por ejemplo, la mayor parte de los cambios efectuados en las legislaciones agrarias y civiles ha surgido como respuesta a las presiones del movimiento feminista internacional y a los compromisos internacionales adquiridos por los Estados, tales como la CEDAW (Deere \& León, 1997, p. 25).

En Colombia, la Asociación Nacional de Mujeres Campesinas e Indígenas de Colombia y la Mesa de Incidencia de Mujeres Rurales Colombianas fueron actores clave para el diseño de la Ley 731, así como 
de los planes y programas que afectan a las mujeres rurales (Mesa de Incidencia de Mujeres Rurales Colombiana, 2015). Sin embargo, un informe presentado por Oxfam indica la falta de estrategias para incorporar las visiones de las mujeres y las organizaciones sociales en el diseño y planificación del Programa de Mujer Rural, sobre todo en lo que tiene que ver con su ejecución a nivel territorial (Vargas \& Villarreal, 2014).

A través de esta ley se permitió la participación de las mujeres en "todas las entidades y órganos de decisión del orden nacional, departamental y municipal, que realicen políticas, planes, programas o proyectos o creen medidas encaminadas a favorecer el sector rural" (Artículo 20), así como en los órganos de decisión de los consejos comunitarios y en las juntas departamentales, distritales y municipales de educación. Asimismo, creó el Comité Interinstitucional de Seguimiento de la Ley, en el que deben participar representantes de las mujeres rurales; sin embargo, este empezó a funcionar solo hasta 2010 y con alcances limitados que no han sido superados en la actualidad (ver sección sobre metas e indicadores).

\section{Registros e información desagregada}

Uno de los grandes problemas para hacer análisis de género en el mundo rural, al menos para el caso de América Latina, es la ausencia de información oficial y confiable que permita hacer las evaluaciones de política pública y de los impactos que han tenido en contextos específicos. Deere y León (1997; 2000; 2003), y Meertens (2006) coinciden en señalar los problemas metodológicos que existen a la hora de cuantificar y esclarecer las desigualdades de género en el campo y que, por ende, se convierten en una barrera para hacer diagnósticos precisos sobre los éxitos o fracasos de los programas a nivel local, regional y nacional.

La razón principal es que los registros de información con variables desagregadas por género (y otras categorías) están en una etapa de construcción. Antes de la entrada del nuevo siglo, tener información disponible sobre la distribución de tierras y otros activos por género era una tarea prácticamente imposible. Los registros estatales empezaron a tener en consideración la variable género a finales de la década de los noventa y solo en 2002, con la Ley 731, se estableció la obligación legal de llevar información desagregada. 
Además, dado que la transversalización de género responde a la diferencia, lograr registros diferenciados únicamente por sexo/género no ayuda a revelar las desventajas a las que están sometidas ciertas mujeres, por ejemplo, las campesinas o las indígenas. Las estadísticas desagregadas deben incluir, además de las variables de género, otras que respondan a unidades de análisis más pequeñas (grupo étnico, edad) o variables espaciales (Rees, 1998). La política de restitución es un buen ejemplo de los esfuerzos que desde hace pocos años ha realizado el Estado para recoger información que responda a dichos criterios.

A pesar de las limitaciones de la política (Parada-Hernández \& PeñaHuertas, 2018), el trabajo realizado al interior de la Unidad de Víctimas y la Unidad de Restitución de Tierras ${ }^{8}$ son un ejemplo de los esfuerzos por mejorar los sistemas de registro y por contar con información desagregada que responda al principio de enfoque diferencial que "reconoce que hay poblaciones con características particulares en razón de su edad, género, orientación sexual y situación de discapacidad" (Ley 1448, Artículo 13) y por ello insta al Estado a que las medidas de reparación cuenten con dicho enfoque.

\section{Seguimiento y evaluación}

Los instrumentos de evaluación de las políticas también deben contar con un enfoque que reconozca los problemas a los que se enfrentan las mujeres y establezcan opciones de política que no obedezcan a supuestos basados en estereotipos, ideologías o políticas ciegas a las cuestiones de género (Beveridge \& Nott, 2002). En este sentido, se hace necesario un ejercicio de innovación en las herramientas para hacer políticas, incluyendo el diseño de indicadores de género (Daly, 2005). Estos últimos representan un gran reto en tanto que su diseño requiere criterios uniformes que sean determinados por consenso; asimismo, resulta muy difícil medir los cambios en las relaciones de poder y el estatus social de las mujeres (Moser \& Moser, 2005).

Los documentos de política revisados dan cuenta de la ausencia de metas claras y de indicadores de género frente al cumplimiento de los

8 Creadas mediante Ley 1448 de 2011 o Ley de Víctimas y Restitución de Tierras. 
planes y programas para las mujeres rurales ${ }^{9}$. Esto obedece, principalmente, a las dificultades que representa encontrar registros diferenciados, al menos hasta antes de 2002. Además, varios de los indicadores diseñados para las políticas de desarrollo rural tienen un enfoque "familista" (Muñoz, 2018) en el que se establece como indicador la familia o al grupo familiar. Ejemplo de ello son los programas de titulación de tierras del Incoder, entidad que "(...) no tiene una orientación técnica ni de política para definir que entiende por 'familia o grupo familiar', ni atiende aspectos relacionados con 'la familia' tales como sus dinámicas, transformaciones y dificultades de tipo civil o social (...)" (USAID, 2013). Estos ejercicios impiden dar cuenta del alcance que tienen las políticas, los planes y los programas para las mujeres.

La tarea de seguimiento y monitoreo de las acciones, que desde los distintos entes se adelantarán en beneficio de la equidad y participación de la mujer, ha sido asignada a la Consejería para la Equidad de la $\mathrm{Mu}^{-}$ jer (antes Dirección Nacional para la Equidad de la Mujer, creada por la Ley 188 de 1995). Se encontró que la las estrategias para impulsar la transversalización de género priorizó las siguientes políticas: Banca de Oportunidades, Red Juntos, atención a la población desplazada, lucha contra la trata de personas y política de reinserción y reintegración económica. Solo hasta la firma del acuerdo de paz, que insiste en la incorporación del enfoque de género en la Reforma Rural Integral, la política de mujeres rurales recobró fuerza en la agenda pública y permitió la reactivación de las instancias de seguimiento y evaluación de la Ley 731 .

Concretamente, la ley dispuso de la creación de un Comité Interinstitucional de Seguimiento a la Ley, establecido tan solo hasta 2010 (ocho años después de expedida la ley); y, durante varios años, funcionó desprovisto de un plan de seguimiento que delimitara acciones y responsables del cumplimiento de las disposiciones legales. La Consejería para la Equidad de la Mujer manifestó al Congreso de la República que entre 2002 y 2014 no se encontraron registros de un plan de acción para el seguimiento de la ley, sino acciones aisladas de fomento a la productividad

9 Dentro de los documentos revisados no se incluyeron aquellos desarrollados por cada una de las agencias, en especial después de la expedición de la Ley 731. Esto podría afectar la conclusión que aquí se presenta. 
y al emprendimiento de las mujeres rurales (Alta Consejería Presidencial para la Equidad de la Mujer, 2016). En 2017, se expidió el Decreto 2145 que reglamentó su funcionamiento y estableció los lineamientos para la creación del Plan de Revisión, Evaluación y Seguimiento de los Programas. No obstante, el plan no ha sido aprobado y continúa en la etapa de diseño (Muñoz, 2018).

\section{Burocracias capacitadas}

Algunos análisis demuestran la importancia que tienen los niveles medios y altos de la administración burocrática en la construcción de las representaciones de género y en la formación de las expectativas ciudadanas frente a los objetivos que se desea perseguir (a través de la redacción de documentos, la manipulación de los significados, la distribución de poderes a los individuos) (Stratigaki, 2005). Así las cosas, al interior de las burocracias los conceptos de género están más expuestos a la cooptación y por ello requieren una mayor atención (Clisby \& Enderstein, 2017).

De ahí la importancia de permitir que se amplíe la participación de mujeres y de personal con conocimiento técnico en temas de género en los distintos niveles de la administración. Además, existe evidencia que muestra que en los países con una mayor presencia de mujeres en posiciones de poder dentro del Estado, y por tanto con un mayor nivel de democracia, más alta es la probabilidad de que un gobierno adopte la estrategia de la transversalización de género (True \& Mintrom, 2001; Squires, 2005).

Para el caso colombiano, Sañudo (2015) muestra la importancia de contar con mujeres con alto poder de decisión en el Estado y preocupadas por avanzar en una agenda proequidad. Estas, a las que ella llama femócratas, fueron cruciales en la construcción de la política pública de mujer rural en Colombia. Asimismo, instancias como la Alta Consejería para la Equidad de la Mujer y la Dirección de Mujer Rural (creada en 2015), ambas encabezadas por mujeres, se han convertido en espacios importantes para dar continuidad a la política pública de mujer rural (Consejería Presidencial para la Equidad de la Mujer \& Dirección de Mujer Rural, 2017; Dirección de Mujer Rural, 2017). 
Fortalecer estas instancias se convierte en una oportunidad para superar la alta discrecionalidad de los funcionarios públicos en cuanto a la posibilidad de no acoger las decisiones de política que benefician a las mujeres rurales (Mesa de Incidencia de Mujeres Rurales Colombiana, 2015; León \& Deere, 1997; Parada-Hernández \& Peña-Huertas, 2018).

\section{Reflexiones}

Son distintas las formas en las que los Estados y las organizaciones internacionales han abordado el problema de la equidad de género. En principio se promovieron estrategias que buscaron la igualdad formal entre hombres y mujeres a través de la trasformación de las normas formales y del reconocimiento de los derechos de las mujeres; posteriormente, se hizo una apuesta por crear mecanismos de inclusión específicos para las mujeres como una medida transitoria de inclusión; finalmente, se propuso una estrategia más amplia de incorporar el género en todos los sistemas y procesos de política, haciendo énfasis en la necesidad de incluir a las mujeres en la toma de decisiones.

Los tres enfoques han aportado herramientas indispensables para cumplir con el objetivo de disminuir las desigualdades en razón del género, aunque la transversalización de género, que incorpora los otros dos enfoques, se ha impuesto como una prioridad en las agendas estatales y de los organismos internacionales. Sin embargo, el proceso de adoptar este enfoque ha estado marcado por el ensayo y error, y nos muestra que queda un largo camino para afinar el contenido del concepto y determinar cuáles son los mecanismos más idóneos para cumplir con los objetivos últimos del enfoque.

Para el caso colombiano, la transversalización de género es un concepto en desarrollo que, además, se enfrenta a un contexto de transformación institucional amparado por un acuerdo de paz. El escenario de posacuerdo se muestra como una ventana de oportunidad para repensar los diseños de política con una mirada de género. La Reforma Rural Integral y la Política Nacional de Sustitución de Cultivos Ilícitos (puntos 1 y 4 del acuerdo) son ahora un marco de acción para implementar este enfoque 
y aquí la sociedad colombiana debe apostar por la transformación de los procesos que, tradicionalmente, han excluido a las mujeres rurales.

El Estado colombiano, si bien ha mostrado interés en aplicar el enfoque, se ha concentrado en promover mecanismos de acción positiva a través de la creación de leyes y programas orientados a atender de manera preferencial a las mujeres; en muchos de los casos se han promovido medidas enfocadas en cierto tipo de mujeres (cabeza de hogar, víctimas del conflicto y mujeres rurales). Como se mostró en el texto, estas medidas son importantes para la inclusión de las mujeres en diferentes escenarios de la vida social; sin embargo, resultan insuficientes para combatir las desigualdades de género en tanto que dejan de ser medidas provisionales para convertirse en la norma general y se aplican sin un análisis profundo sobre cómo opera la desigualdad en los contextos locales.

Las respuestas del Estado colombiano a las demandas de las mujeres rurales son un ejemplo del camino que hace falta recorrer para transversalizar el género en la política de desarrollo rural. Como se mostró, las iniciativas promovidas a favor de las mujeres rurales hacen parte de una agenda paralela a la del desarrollo rural en su conjunto. Así las cosas, la intención de transformar los procesos por medio de los cuales se diseñan políticas públicas se queda corta al momento de crear mecanismos para garantizar la participación de las mujeres en dicho diseño, pues se las aísla en sus propios procesos de política enteramente diferenciada y al momento de abordar las desigualdades de género en espacios como el rural.

Las barreras que persisten en este tratamiento diferenciado de las mujeres rurales, y que fueron identificadas en el texto, son un indicio de los problemas que tiene el Estado colombiano al momento de dotarse de herramientas para cumplir con los objetivos que se propone. En este caso, los compromisos de género y la redistribución de capitales entre hombres y mujeres se ven minados por: (i) vacíos informacionales que se traducen en la incomprensión del problema de la distribución de activos y de las dinámicas propias del mundo rural; (ii) los órdenes de género que aún subsisten en los diseños institucionales y que tienen como consecuencia la reproducción de estereotipos que promueven la desigualdad; y (iii) la creación de programas sin el fortalecimiento de capacidades del Estado que garanticen la sostenibilidad de los programas a largo plazo. 
La transversalización de género, para que sea efectiva, no puede perseguirse únicamente mediante mecanismos de reconocimiento formal de derechos o de acción positiva. Esta requiere de una comprensión de cómo ciertos fenómenos afectan, de manera específica, a las mujeres y, a partir de ello, transformar las instituciones políticas, sociales y económicas que mantienen la redistribución inequitativa de recursos y de poder entre hombres y mujeres.

\section{Referencias}

Alta Consejería Presidencial para la Equidad de la Mujer. (2012). Lineamientos de la política pública nacional para la equidad de género. Bogotá: Alta Consejería Presidencial para la Equidad de la Mujer.

Alta Consejería Presidencial para la Equidad de la Mujer. (2016). Informe Ley 731. Audiencia Pública de seguimiento a la Ley 731. Bogotá: Congreso de la República.

Asamblea General de las Naciones Unidas. (1979). Convención sobre la Eliminación de Todas las Formas de Discriminación contra la Mujer. Nueva York.

Beveridge, F., E Nott, S. (2002). Mainstreaming: A case for optimism and cynicism. Feminist legal studies, (10), 299-311.

Clisby, S., \& Enderstein, A. M. (2017). Caught between the orientalist-occidentalist polemic: Gender mainstreaming as feminist transformation or neocolonial subversion? International Feminist Journal of Politics, 19(2), 231-246.

Cockburn. (1991). In the way of women: Men's resistance to sex equality in organisations. Londres: Macmillan.

Consejería Presidencial para la Equidad de la Mujer \& Dirección de Mujer Rural. (2017). Plan de revisión, evaluación y seguimiento de los programas que favorecen a las mujeres rurales. Bogotá, D. C: Presidencia de la República.

Dahlerup, D. (2006). Women, quotas and politics. Nueva York: Routledge.

Daly, M. (2005). Gender mainstreaming in theory and practice. Social Politics: International Studies in Gender, State and Society, 12(3), 433-450.

Deere, C. D., E León, M. (2000). Género, propiedad y empoderamiento: tierra, Estado y mercado en América Latina. Bogotá: Editores Tercer Mundo; Universidad Nacional de Colombia.

Deere, C., E León, M. (2003). The gender asset gap: Land in Latin America. World Development, 31(6), 925-947. 
Dirección de Mujer Rural. (2017). Plan Estratégico de la Dirección de la Mujer Rural 2017-2020. Bogotá: Ministerio de Agricultura y Desarrollo Rural.

Durango Álvarez, G. (2016). Las acciones afirmativas como mecanismos reivindicadores de la paridad de género en la participación política inclusiva: Ecuador, Bolivia, Costa Rica y Colombia. Revista de Derecho, (45), 137-168. Gouges, O. D. (1791). Declaración de los Derechos de la Mujer y de la Ciudadana. Tomado de: https://www.nodo50.org/xarxafeministapv/IMG/pdf/declaracionDerechosMujer.pdf

Guerrina, R. (2002). Mothering in Europe: Feminist critique of European policies on motherhood and employment. European Journal of Women's Studies, 9(1), 49-68.

Hafner-Burton, E., E Pollack, M. (2002). Gender mainstreaming and global governance. Feminist Legal Studies, (10), 285-298.

Hankivsky, O. (2005). Gender vs. diversity mainstreaming: A preliminary examination of the role and transformative potential of feminist theory. Canadian Journal of Political Science, 38(4), 977-1001.

Jaramillo, I. C. (2000). La crítica feminista al derecho. In R. West, Género y teoría del derecho (pp. 27-66). Bogotá: Siglo del Hombre.

Kabeer, N. (2003). Institutionalising gender equity goals in the policy process. In I. D. Centre, Gender mainstreaming in poverty eradication and the millennium development goals: A handbook for policy-makers and other stakeholders, (pp. 197-233). Toronto: Commonwealth Secretariat, CIDA, IDRC, Canadian International Development Agency.

Klein, A. (2012). Slipping racism into the mainstream: A theory of information laundering. Communication Theory, 22(4), 427-448.

León, M., E Deere, C. D. (1997). La mujer rural y la reforma agraria en Colombia. Cuadernos de Desarrollo Rural, (38-39), 7-23.

Mazey, S. (2002). Gender mainstreaming strategies in the EU: Delivering on an agenda? Feminist Legal Studies, (10), 227-240.

Meertens, D. (2006). Tierra, derechos y género. Leyes, politicas y prácticas en contextos de guerra y paz. Bogotá: Fondo de Desarrollo de las Naciones Unidas para la Mujer.

Mehra, R., E Gupta, G. (2006). Gender mainstreaming: Making it happen. Washington, D. C.: Rep. International Center for Research on Women.

Mesa de Incidencia de Mujeres Rurales Colombianas. (2015). La tierra prometida. La vida después de la guerra. Bogotá. Tomado de http://cjyiracastro.org.co/ mujeres-opinan/la-tierra-prometida-la-vida-despues-de-la-guerra/

Morris, C. E Little, J. (2005). Critical studies in rural gender issues. Farnham: Ashgate. 
Moser, C., \& Moser, A. (2005). Gender mainstreaming since Beijing: A review of success and limitations in international institutions. Gender $\mathcal{E}$ Development, 13(2), 11-22.

Mouritz Marfelt, M. (2006). Grounded intersectionality. Equality, Diversity and Inclusion: An International Journal, 35(1), 31- 47.

Muñoz, G. D. (2018, febrero 19). Consejería Presidencial para la Equidad de la Mujer. (M. Parada, entrevistadora).

Murillo Martínez, P. E. (2010). Las medidas de acción afirmativa o medidas especiales: para reparar las injusticias históricas y la discriminación. El Otro Derecho, (41).

Mwagiru, M. (2001). Women's land and property rights in three Eastern Africa countries. En A. Buregeya, M, Garling, J. Craig \& B. Harrell-Bond (Eds). Women's land and property rights in situations of conflict and reconstruction: A reader based on the February, 1998 Inter-Regional Consultation in Kigali, Rwanda, (pp. 18-23). Nueva York: United Nations Development Fund for Women.

Ospina Robledo, R. I. (1998). Misión rural. Para empoderar a las mujeres rurales. Bogotá: IICA \& TM Editores.

Parada-Hernández, M. M. E Peña-Huertas, R. D. (2018). Avances y talanqueras del enfoque diferencial de género en la restitución de tierras. Bogotá: Universidad del Rosario, (en prensa).

Pasura, D. (2010). A gendered analysis of land reforms in Zimbabwe. Women's Studies International Forum, 33(5), 443-454.

Rees, T. (1998). Mainstreaming equality in the European Union. Education, training and labour market policie. Londres: Routledge.

Saldarriaga Grisales, D. C. E Ramírez Monsalve, P. A. (2015). Acciones afirmativas. Políticas en pugna con la discriminación que develan estructuras hegemónicas de sostenimiento. Ratio Juris, 10(20), 115-138.

Sañudo, M. F. (2015). Tierra y género. Bogotá: Universidad Javeriana.

Squires, J. (2005). Is mainstreaming transformative? Theorizing mainstreaming in the context of diversity and deliberation. Social Politics: International Studies in Gender, State E Society, 12(3), 366-388.

Stratigaki, M. (2005). Gender mainstreaming vs. positive action: An ongoing conflict in EU gender equality policy. European Journal of Women's Studies, 12(2), 165-186.

True, J. E Mintrom, M. (2001). Transnational networks and policy diffusion: The case of gender mainstreaming. International Studies Quarterly, (45), 27-57.

USAID. (2013). Informe final. Programa de políticas públicas de USAID. Bogotá, D. C.: USAID. 
Vargas, C. E Villarreal, N. (2014). Programa Mujer Rural, avances, obstáculos y desafíos. Bogotá: Oxfam Colombia.

Verloo, M. (2001). Another velver revolution? Gender mainstreaming and the politis of implementation. Vienna: Institute fo Human Sciences.

Walby, S. (2005). Gender mainstreaming: Productive tensions in theory and practice. Social Politics: International Studies in Gender, State E Society, 12(3), 321-343.

Walker, C. (2003). Piety in the sky? Gender policy and land reform in South Africa. Journal of Agrarian Change, 3(1-2), 113-148.

Wollstonecraft, M. (1972). A vindication of the righst of woman. Londres: Penguin Books.

Yeandre, S., Booth, C. E Bennet, C. (1998). Criteria for success of a manistreming approach to gender equality. Sheffield: CRESR. 